\title{
Detection of CRB1 mutations in families with retinal dystrophy through phenotype-oriented mutational screening
}

\author{
SHIQIANG LI ${ }^{*}$, TAO SHEN ${ }^{*}$, XUESHAN XIAO, XIANGMING GUO and QINGJIONG ZHANG \\ State Key Laboratory of Ophthalmology, Zhongshan Ophthalmic Center, \\ Sun Yat-sen University, Guangzhou, Guangdong 510060, P.R. China
}

Received September 17, 2013; Accepted January 28, 2014

DOI: $10.3892 /$ ijmm.2014.1655

\begin{abstract}
Mutations in the crumbs homolog (CRB)1 gene are among the common causes of severe early onset retinal dystrophy. Some characteristic clinical phenotypes are frequently associated with mutations in CRB1. The aim of this study was to examine whether characteristic phenotypedirected mutational screening facilitated the detection of CRB1 mutations. The study included 22 probands with at least one of the potential CRB1-associated phenotypes for retinal dystrophy. Variants were detected using Sanger sequencing. The complete sequences of the coding and adjacent intronic regions of CRB1 were analyzed, revealing homozygous or compound heterozygous mutations in CRB1 in seven of 22 probands, involving six novel (c.136delA, c.1841G > T, c.3017C >A, c. $3488 \mathrm{G}>\mathrm{T}$, c. $3991 \mathrm{C}>\mathrm{T}$ and c.4089dupTGTTGCTT) and four known (c.2222T $>$ C, c. $2671 \mathrm{~T}>\mathrm{G}$, c. $3676 \mathrm{G}>\mathrm{T}$ and c. $4005+2 \mathrm{~T}>\mathrm{G})$ mutations. The mutations were present in three of four probands with macular nummular pigmentation and in four of seven probands with early onset retinitis pigmentosa with macular involvement. The results suggested that macular nummular pigmentation is a gene-specific indication for CRB1-associated retinal dystrophy and confirm that CRB1 mutations are also common causes of early onset retinitis pigmentosa. Identification of gene-specific phenotypes is uselful in identifying genetic defects underlying heterogeneous retinal dystrophy.
\end{abstract}

\section{Introduction}

Hereditary retinal dystrophy is a broad group of clinically and genetically heterogeneous diseases that usually cause

Correspondence to: Dr Qingjiong Zhang, Zhongshan Ophthalmic Center, Sun Yat-sen University, 54 Xianlie Road, Guangzhou, Guangdong 510060, P.R. China

E-mail: zhangqji@mail.sysu.edu.cn; qingjiongzhang@yahoo.com

*Contributed equally

Key words: crumbs homolog 1, novel mutation, phenotype-oriented, retinal dystrophy irreversible blindness. Retinitis pigmentosa (RP) is the most common form, with Leber congenital amaurosis (LCA) being the most severe form. The clinical manifestations as well as the causative genes frequently overlap in the different forms of these diseases (1). Mutations in almost 200 genes have been described in association with hereditary retinal diseases (RetNet: http://www.sph.uth.tmc.edu/Retnet/sum-dis.htm). However, mutations in each of these genes usually contribute to only a small fraction of the diseases (2-4). Thus, detection of disease-causing mutations among these genes is a great challenge in individual patients, and involves great effort and expense. Identification of a gene-specific genotype-phenotype correlation would facilitate mutation detection, similar to CYP4V2 mutations and Bietti crystalline corneoretinal dystrophy $(5,6)$.

Mutations in crumbs homolog (CRB)1 are among the common causes of severe early onset retinal dystrophy, including LCA and early onset RP (4,7-31). Although a firm genotype-phenotype correlation between CRB1 mutations and specific phenotypes has yet to be determined, some characteristic clinical findings have been suggested to associate with CRB1 mutations.

The aim of the present study was to investigate whether characteristic phenotype-directed mutational screening facilitated the detection of CRB1 mutations. Probands with some of the CRB1-associated phenotypes were selected for this study. The Sanger sequencing of CRB1 coding and adjacent intronic regions revealed homozygous and compound heterozygous mutations in CRB1 in seven of 22 Chinese probands with at least one of the CRB1-associated phenotypes.

\section{Materials and methods}

Clinical characteristics of the probands. Probands from 22 unrelated families with at least one of the CRB1-associated phenotypes participated in this study (Table I). Clinical phenotypes in this study were mainly based on the fundus photos and the age of onset (1-10 years of age). CRB1-associated characteristic clinical findings included: i) severe early onset retinal dystrophy (including LCA and early onset RP) (4,7-31); ii) nummular pigmentary changes at the posterior fundus; iii) preservation of the para-arteriolar retinal pigment epithelium (PPRPE) (14); iv) Coats-like vasculopathy (CLV) (12); and v) pigmented paravenous chorioretinal atrophy (PPCRA) (20). 
Table I. Classification of the probands in this study.

No. of cases

Clinical characteristics

Screened

CRB1 mutations

A. Macular nummular pigmentation

$\begin{array}{rr}4 & 3 \\ 4 & 0 \\ 7 & 4 \\ 7 & 0 \\ 22 & 7\end{array}$

The LCA, PPRPE and CLV phenotypes were not studied since LCA has been previously analyzed, whereas PPRPE and CLV were not identified in our patients. Macular dot pigmentation in seven probands was also selected to examine the specificity of macular nummular pigmentation.

Informed consent was obtained from each participating individual or their guardians prior to the collection of clinical data and venous blood. This study was approved by the Institutional Review Board of the Zhongshan Ophthalmic Center and followed the tenets of the Declaration of Helsinki.

Genomic DNA. Genomic DNA was prepared from venous leukocytes using a method described previously (32). The procedure for Sanger sequencing of CRB1 was the same as that described in our previous study and the same sets of primers were used (4). The informatics analysis for the detected variants was also as described previously (4). Only homozygous or compound heterozygous variants that were predicted to be pathogenic were considered to be disease-causing because: i) CRB1 mutations were only reported to associate with recessive retinal dystrophy (8); and ii) single heterozygous null mutations for recessive genes were common in the general population (33). Controls were healthy university students with normal visual acuity without any known hereditary retinopathy and family history of such diseases as those controls used in the genetic study of myopia (34).

\section{Results}

Homozygous or compound heterozygous mutations in $C R B 1$. Complete sequencing analysis of CRB1 revealed homozygous and compound heterozygous variants in seven of the 22 probands (Fig. 1), involving six novel and four known variations (Table II). Of the 10 variants, four were predicted to be truncated and the remaining six variants were missense. The residues with the six missense changes were relatively conserved in different species (Table II and Fig. 2) and involved in the functional domain of the CRB1 protein (Table II). Five of the six missense variants were predicted to affect the encoded protein by PolyPhen-2, while two variants were predicted to be damaging by sorting intolerant from tolerant (SIFT) (Table II). Only the missense change p.M741T, was predicted to be benign or tolerated by PolyPhen-2 or SIFT, but it was reported to be causative in a previous study (31). Therefore, all 10 variants detected in this study were considered to be pathogenic.
Clinical data of the seven probands with CRB1 mutations. Each proband had either poor vision or nystagmus at $<8$ years of age. Visual acuity ranged from finger counting to 0.6 (Table III). Homozygous or compound heterozygous mutations were identified in three of four probands with macular nummular pigmentation and in four of seven probands with early onset RP with macular involvement. However, no mutations were identified in the 11 probands with either pigmented paravenous chorioretinal atrophy or other retinal dystrophy with macular dot pigmentation (Tables I and III). All seven probands with CRB1 mutations were isolated cases (Fig. 1). Fig. 3 shows the representative fundus changes of three probands with CRB1 mutations (Fig. 3A-C) and one proband without CRB1 mutation (Fig. 3D) but with nummular pigmentation.

\section{Discussion}

A recent review on CRB1 mutations suggested an average prevalence of homozygous or compound heterozygous CRB1 mutations in patients with different forms of retinal dystrophy as follows: $6.6 \%(109 / 1,645)$ for LCA or early onset retinal dystrophy, $1.2 \%$ (4/335) for RP, $66.7 \%$ (18/27) for RP with PPRPE and $26.7 \%$ (8/30) for RP with CLV (8). Those findings suggested that the sequencing analysis of patients with CRB1-specific phenotypes may facilitate mutation detection of CRB1-associated retinopathy. The frequency of CRB1 mutations in patients with macular nummular pigmentation was not available due to the unavailability of the frequency of this fundus change in patients analyzed in previous studies. However, macular nummular pigmentation is rarely observed in patients with mutations in other genes, although it has been described in different studies in a number of patients with CRB1 mutations (9,16,24,25,35-37). Therefore, it may be considered a characteristic indication for CRB1-associated retinal dystrophy.

In the present study, the entire coding region and adjacent intronic sequences of CRB1 were analyzed in 22 probands with one of the possible CRB1-associated phenotypes, using Sanger sequencing. Homozygous and compound heterozygous mutations in the CRB1 gene, involving six novel and four known variants, were identified in $31.8 \%(7 / 22)$ of the probands, including $75 \%$ (3/4) with macular nummular pigmentation and $57.2 \%$ (4/7) with early onset RP with macular involvement. No novel mutations were detected in 192 alleles of 96 healthy individuals. The frequent association of CRB1 mutations with early onset of RP and LCA 

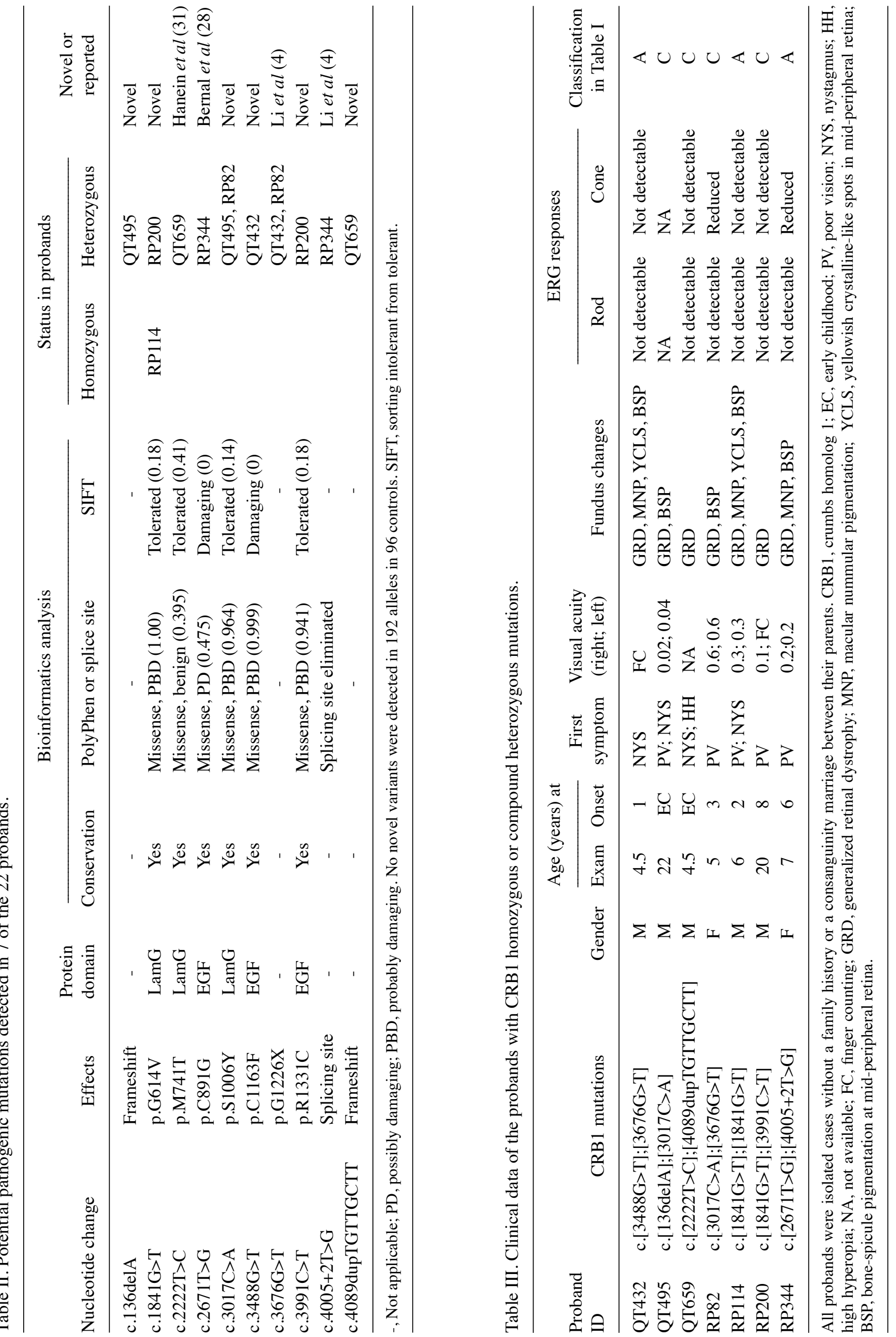

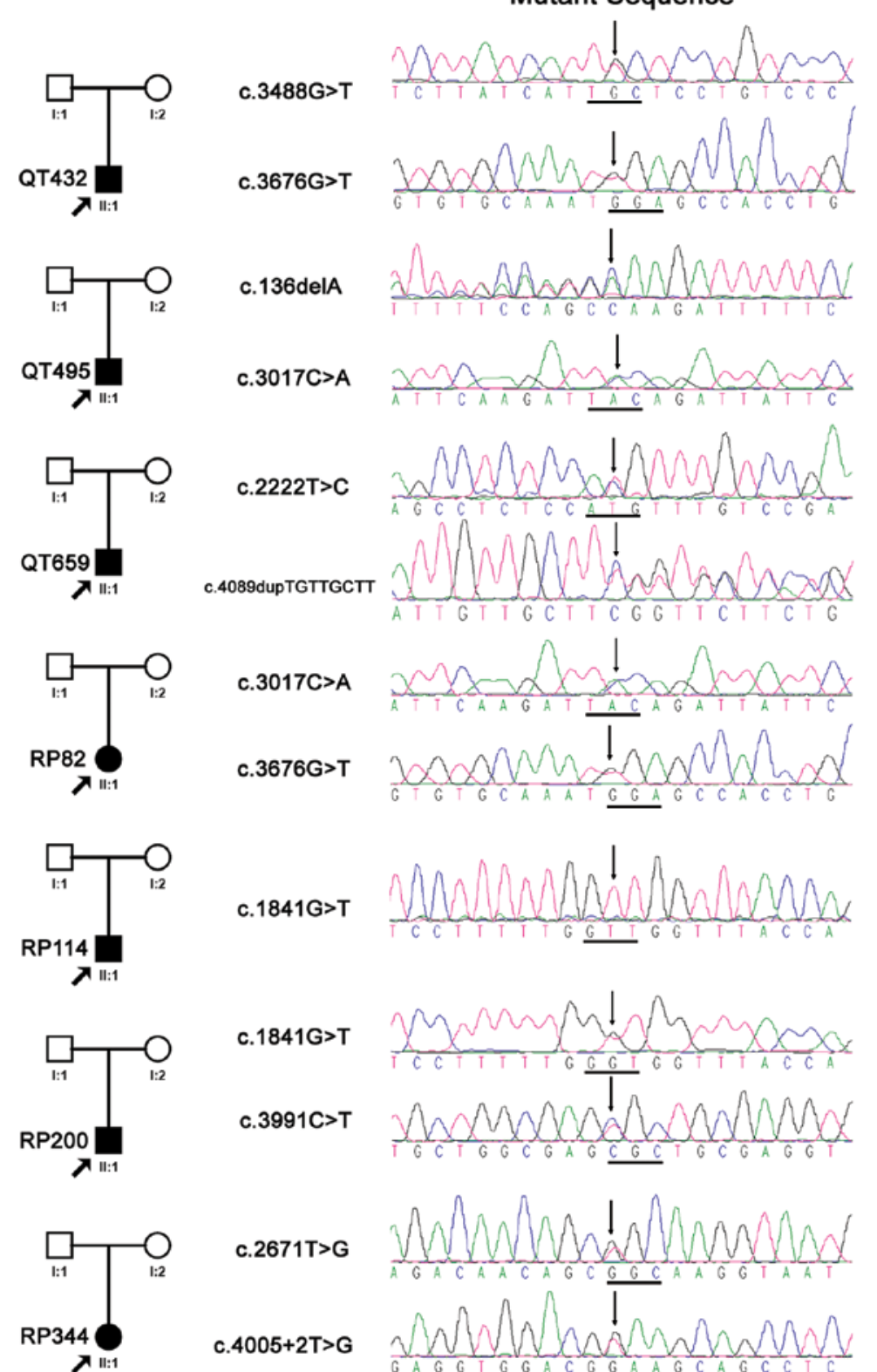

c. $2671 \mathrm{~T}>\mathrm{G}$
Mutant Sequence
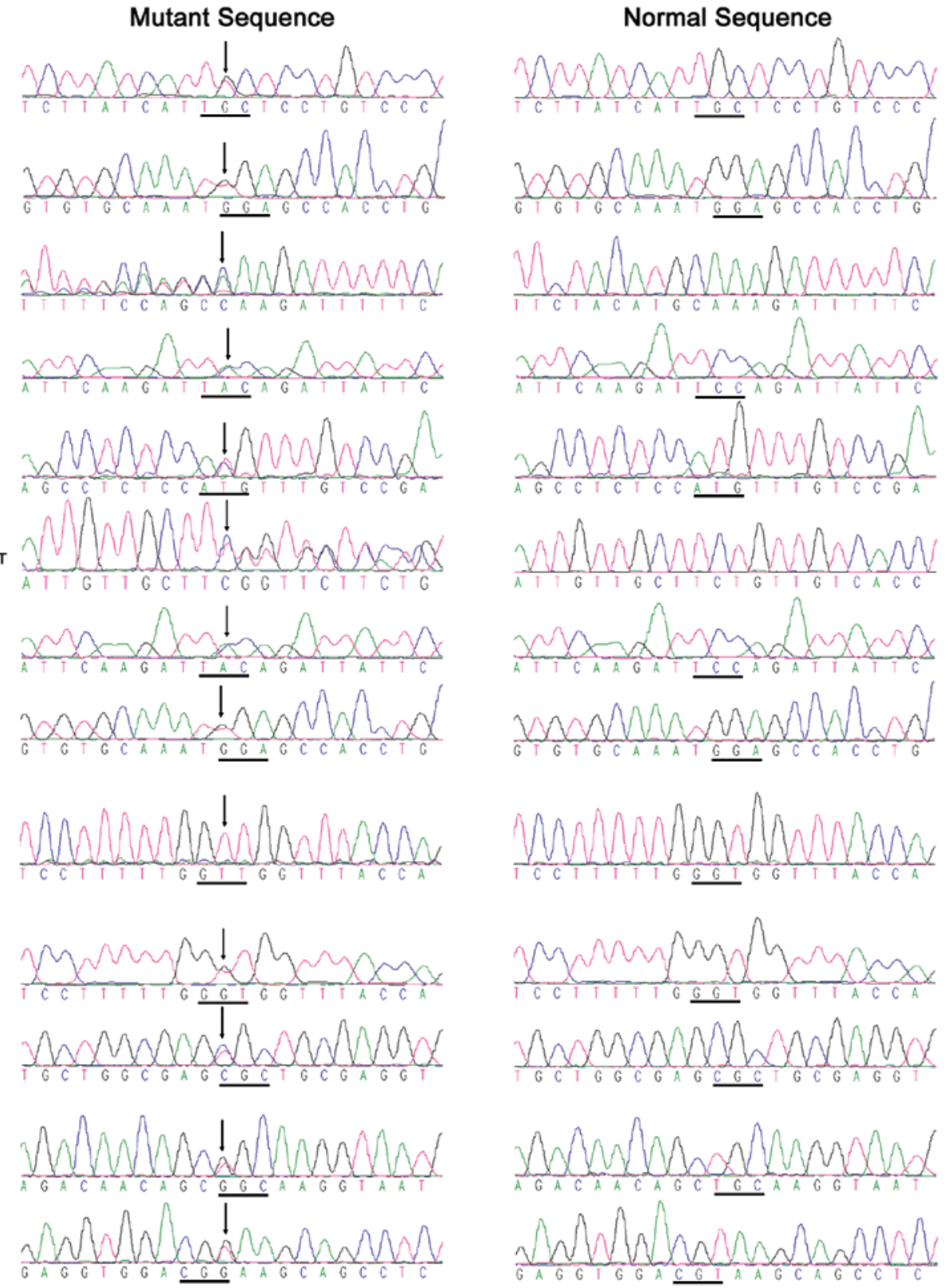

Figure 1. Sequence chromatography. Homozygous or compound heterozygous mutations in crumbs homolog (CRB)1 of each of the seven probands are shown on the left; the corresponding normal sequences are shown on the right.

suggests that early onset RP is a spectrum from LCA to RP with early onset RP being detected within this spectrum. Thus, genes associated with early onset RP are potentially good candidates for LCA and vice versa.

Results of the bioinformatics analysis suggest that these mutations are likely to exert pathogenic effects on the encoded proteins (Fig. 2). Our study provides additional evidence that macular nummular pigmentation may be considered a gene-specific indication for CRB1-associated retinal dystrophy. The analysis also suggests that CRB1 mutations are common causes for early onset RP, as observed by their contribution to LCA. Findings of a recent study also showed that CRB1 mutations are a relatively common cause of autosomal recessive early onset retinal degeneration (38), suggesting that certain CRB1-associated specific phenotypes, including PPRPE and CLV, may not be common in the studied populations. This may also be due to the relatively small sample size of this study.

Identification of gene-specific phenotypes may facilitate mutation identification and subsequent genetic counseling as well as provide useful evidence to determine causative variants underlying the extremely heterogeneous hereditary retinal diseases. This is particularly true for isolated patients with mutations in multiple genes, which are likely to become increasingly common in the era of next-generation sequencing. Systematic and careful records of clinical data, particularly of fundus photos of different regions and at different time-points, may provide additional gene-specific phenotypes for CRB1 and for other genes associated with retinal dystrophy. This record keeping should be encouraged in future investigations of mutations related to retinal dystrophy. 


\begin{tabular}{|c|c|c|c|c|c|c|}
\hline \multirow{10}{*}{$\begin{array}{l}\text { Homo sapiens } \\
\text { Pan troglodytes } \\
\text { Gorilla gorilla gorilla } \\
\text { Macaca mulatta } \\
\text { Rattus norvegicus } \\
\text { Bos taurus } \\
\text { Felis catus } \\
\text { Canis lupus familiaris } \\
\text { Danio rerio } \\
\text { Gallus gallus }\end{array}$} & \multicolumn{6}{|c|}{$\begin{array}{c}\mathrm{C} 1163 \mathrm{~F} \\
\mid\end{array}$} \\
\hline & & $S Y$ & $\mathrm{HC}$ & C s & C & 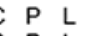 \\
\hline & $Y S$ & $S Y$ & & & & 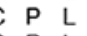 \\
\hline & $Y S$ & $S Y$ & $\mathrm{H} \mathrm{C}$ & & & 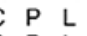 \\
\hline & Y S & & & & & \\
\hline & Y S & & $\mathrm{R}$ & & & \\
\hline & $\mathrm{Y} S$ & & & & & \\
\hline & $Y S$ & & $\mathrm{H}$ & & & \\
\hline & $\mathrm{Y} S$ & & & & & \\
\hline & & & $\mathrm{H}$ & & & $P$ \\
\hline
\end{tabular}

M741T

Homo sapiens

Pan troglodytes

Gorilla gorilla gorilla

Macaca mulatta

Rattus norvegicus

Bos taurus

Felis catus

Canis lupus familiaris

Danio rerio

Gallus gallus
Homo sapiens Pan troglodytes

Gorilla gorilla gorilla Macaca mulatta

Rattus norvegicus

Bos taurus

Felis catus

Canis lupus familiaris

Danio rerio

Gallus gallus

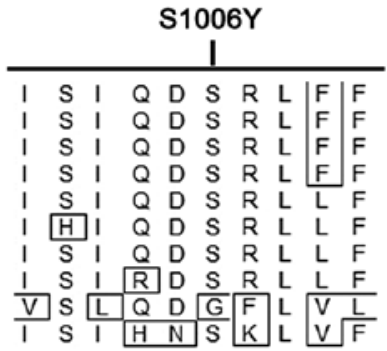

\begin{tabular}{llllllllll}
\multicolumn{10}{c}{$G 614 V$} \\
\hline$Q$ & $N$ & $S$ & $F$ & $L$ & $G$ & $G$ & $L$ & $P$ & $V$ \\
$Q$ & $N$ & $S$ & $F$ & $L$ & $G$ & $G$ & $L$ & $P$ & $V$ \\
$Q$ & $N$ & $S$ & $F$ & $L$ & $G$ & $G$ & $L$ & $P$ & $V$ \\
$Q$ & $N$ & $S$ & $F$ & $L$ & $G$ & $G$ & $L$ & $P$ & $V$ \\
$Q$ & $N$ & $S$ & $F$ & $L$ & $G$ & $G$ & $L$ & $P$ & $M$ \\
$Q$ & $N$ & $S$ & $F$ & $L$ & $G$ & $G$ & $L$ & $P$ & $E$ \\
$Q$ & $N$ & $S$ & $F$ & $L$ & $G$ & $G$ & $L$ & $P$ & $V$ \\
$Q$ & $N$ & $S$ & $F$ & $L$ & $G$ & $G$ & $L$ & $P$ & $G$ \\
\hline$L$ & $S$ & $T$ & $F$ & $V$ & $G$ & $G$ & $L$ & $D$ & $E$ \\
\hline$Q$ & $S$ & $T$ & $F$ & $L$ & $G$ & $S$ & $L$ & $P$ & $V$
\end{tabular}

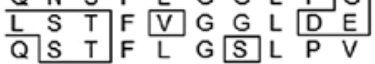

\section{R1331C}

Homo sapiens

Pan troglodytes Gorilla gorilla gorilla Macaca mulatta Rattus norvegicus Bos taurus Felis catus Canis lupus familiaris Danio rerio Gallus gallus

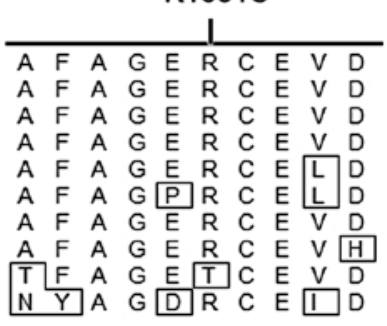

Homo sapiens Pan troglodytes Gorilla gorilla gorilla Macaca mulatta Rattus norvegicus Bos taurus Felis catus Canis lupus familiaris Danio rerio Gallus gallus
Homo sapiens Pan troglodytes Gorilla gorilla gorilla Macaca mulatta Rattus norvegicus Bos taurus

Felis catus Canis lupus familiaris Danio rerio Gallus gallus

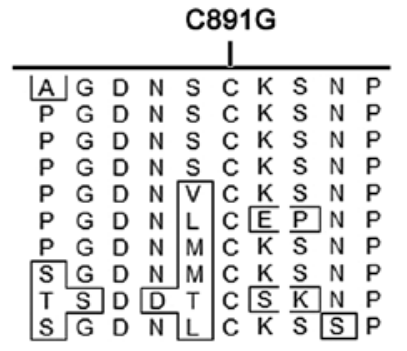

Figure 2. Protein sequence alignment for 10 crumbs homolog (CRB)1 orthologs. Of the six residues affected by the six missense mutations, four are well conserved through all 10 species while the other two are relatively conserved in nine of the ten species. The squares indicate residues that were not conserved in specific species.
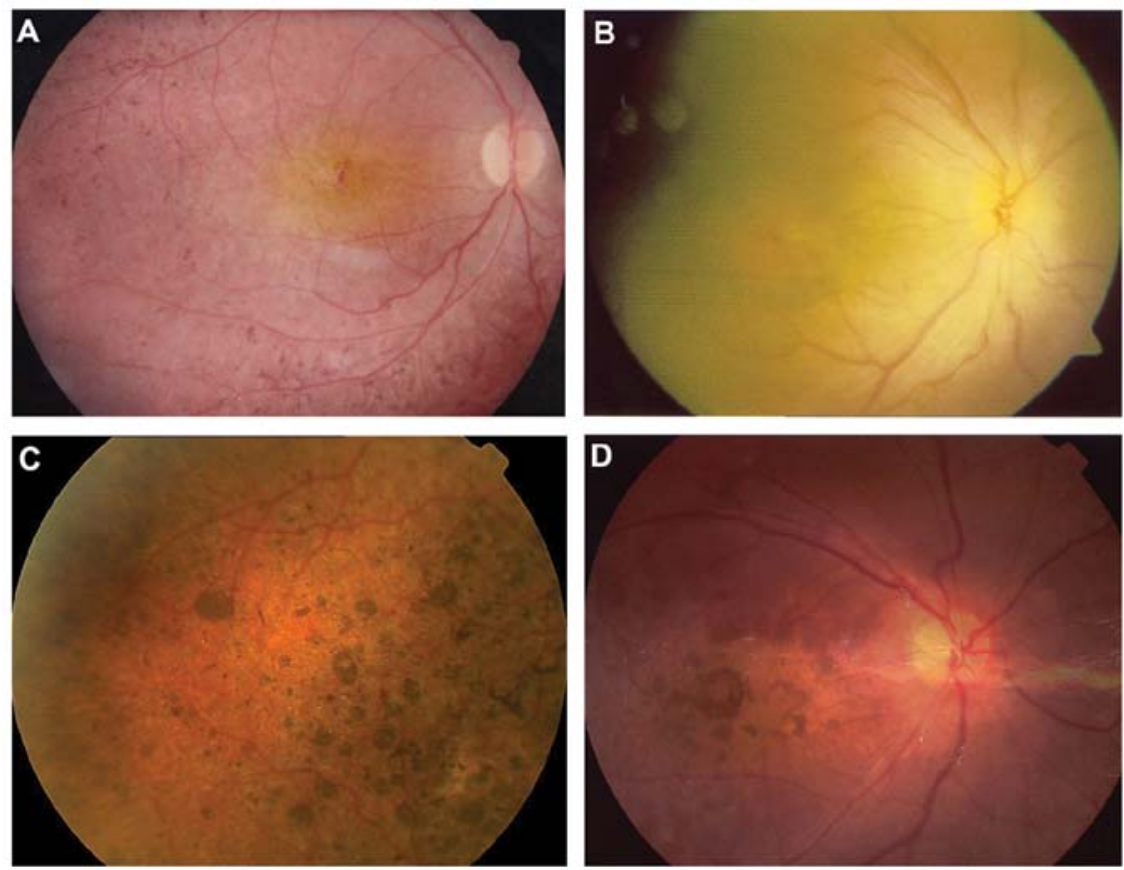

Figure 3. Fundus photos: (A) Generalized retinal dystrophy with early macular involvement and bone-spicule pigmentation in mid-peripheral retina, which was obtained from proband QT495 with crumbs homolog (CRB)1 compound heterozygous mutations (c.[136delA]; [3017C >A]). (B) Generalized retinal dystrophy with early macular involvement, which was obtained from proband QT659 with CRB1 compound heterozygous mutations (c.[2222T>C; [4089dupTGTTGCTT]). (C) The sample was obtained from proband RP114 with homozygous CRB1 mutations (c.[1841G>T]; [1841G>T]), and indicates generalized retinal dystrophy, nummular pigmentation, and yellowish crystalline-like spots. (D) Only proband with nummular pigmentation but without CRB1 mutations was identified. 


\section{Acknowledgements}

The authors would like to thank the patients for their participation and the anonymous reviewers for their constructive advice. This study was supported by the NSFC81170881 (to Q.Z.).

\section{References}

1. Berger W, Kloeckener-Gruissem B and Neidhardt J: The molecular basis of human retinal and vitreoretinal diseases. Prog Retin Eye Res 29: 335-375, 2010.

2. den Hollander AI, Black A, Bennett $\mathrm{J}$ and Cremers FP: Lighting a candle in the dark: advances in genetics and gene therapy of recessive retinal dystrophies. J Clin Invest 120: 3042-3053, 2010.

3. Hartong DT, Berson EL and Dryja TP: Retinitis pigmentosa. Lancet 368: 1795-1809, 2006.

4. Li L, Xiao X, Li S, et al: Detection of variants in 15 genes in 87 unrelated Chinese patients with Leber congenital amaurosis. PLoS One 6: e19458, 2011.

5. Li A, Jiao X, Munier FL, et al: Bietti crystalline corneoretinal dystrophy is caused by mutations in the novel gene CYP4V2. Am J Hum Genet 74: 817-826, 2004.

6. Xiao X, Mai G, Li S, et al: Identification of CYP4V2 mutation in 21 families and overview of mutation spectrum in Bietti crystalline corneoretinal dystrophy. Biochem Biophys Res Commun 409: 181-186, 2011.

7. Corton M, Tatu SD, Avila-Fernandez A, et al: High frequency of CRB1 mutations as cause of early-onset retinal dystrophies in the Spanish population. Orphanet J Rare Dis 8: 20, 2013.

8. Bujakowska K, Audo I, Mohand-Saïd S, et al: CRB1 mutations in inherited retinal dystrophies. Hum Mutat 33: 306-315, 2012.

9. Abouzeid H, Li Y, Maumenee IH, et al: A G1103R mutation in CRB1 is co-inherited with high hyperopia and Leber congenital amaurosis. Ophthalmic Genet 27: 15-20, 2006.

10. Booij JC,Florijn RJ, ten Brink JB, et al: Identification of mutations in the AIPL1, CRB1, GUCY2D, RPE65, and RPGRIP1 genes in patients with juvenile retinitis pigmentosa. J Med Genet 42: e67, 2005.

11. den Hollander AI, Davis J, van der Velde-Visser SD, et al: CRB1 mutation spectrum in inherited retinal dystrophies. Hum Mutat 24: $355-369,2004$

12. den Hollander AI, Heckenlively JR, van den Born LI, et al: Leber congenital amaurosis and retinitis pigmentosa with coats-like exudative vasculopathy are associated with mutations in the crumbs homologue 1 (CRB1) gene. Am J Hum Genet 69: 198-203, 2001.

13. den Hollander AI, Lopez I, Yzer S, et al: Identification of novel mutations in patients with Leber congenital amaurosis and juvenile RP by genome-wide homozygosity mapping with SNP microarrays. Invest Ophthalmol Vis Sci 48: 5690-5698, 2007.

14. den Hollander AI, ten Brink JB, de Kok YJ, et al: Mutations in a human homologue of Drosophila crumbs cause retinitis pigmentosa (RP12). Nat Genet 23: 217-221, 1999.

15. Gerber S, Perrault I, Hanein S, et al: A novel mutation disrupting the cytoplasmic domain of CRB1 in a large consanguineous family of Palestinian origin affected with Leber congenital amaurosis. Ophthalmic Genet 23: 225-235, 2002.

16. Henderson RH, Mackay DS, Li Z, et al: Phenotypic variability in patients with retinal dystrophies due to mutations in CRB1. Br J Ophthalmol 95: 811-817, 2011.

17. Jalkh N, Guissart C, Chouery E, et al: Report of a novel mutation in CRB1 in a Lebanese family presenting retinal dystrophy. Ophthalmic Genet: Jan 30, 2013 (Epub ahead of print).

18. Lotery AJ, Jacobson SG, Fishman GA, et al: Mutations in the CRB1 gene cause Leber congenital amaurosis. Arch Ophthalmol 119: 415-420, 2001.

19. Lotery AJ, Malik A, Shami SA, et al: CRB1 mutations may result in retinitis pigmentosa without para-arteriolar RPE preservation. Ophthalmic Genet 22: 163-169, 2001.
20. McKay GJ, Clarke S, Davis JA, et al: Pigmented paravenous chorioretinal atrophy is associated with a mutation within the crumbs homolog 1 (CRB1) gene. Invest Ophthalmol Vis Sci 46: 322-328, 2005.

21. McMahon TT, Kim LS, Fishman GA, et al: CRB1 gene mutations are associated with keratoconus in patients with leber congenital amaurosis. Invest Ophthalmol Vis Sci 50: 3185-3187, 2009.

22. Paun CC, Pijl BJ, Siemiatkowska AM, et al: A novel crumbs homolog 1 mutation in a family with retinitis pigmentosa, nanophthalmos, and optic disc drusen. Mol Vis 18: 2447-2453, 2012.

23. Riveiro-Alvarez R, Vallespin E, Wilke R, et al: Molecular analysis of ABCA4 and CRB1 genes in a Spanish family segregating both Stargardt disease and autosomal recessive retinitis pigmentosa. Mol Vis 14: 262-267, 2008.

24. Yzer S, Fishman GA, Racine J, et al: CRB1 heterozygotes with regional retinal dysfunction: implications for genetic testing of leber congenital amaurosis. Invest Ophthalmol Vis Sci 47: 3736-3744, 2006.

25. Zenteno JC, Buentello-Volante B, Ayala-Ramirez R and Villanueva-Mendoza C: Homozygosity mapping identifies the Crumbs homologue 1 (Crb1) gene as responsible for a recessive syndrome of retinitis pigmentosa and nanophthalmos. Am J Med Genet A 155A: 1001-1006, 2011.

26. Benayoun L, Spiegel R, Auslender N, et al: Genetic heterogeneity in two consanguineous families segregating early onset retinal degeneration: the pitfalls of homozygosity mapping. Am J Med Genet A 149A: 650-656, 2009.

27. Bustamante-Aragones A, Vallespin E, Rodriguez de Alba M, et al: Early noninvasive prenatal detection of a fetal CRB1 mutation causing Leber congenital amaurosis. Mol Vis 14: 1388-1394, 2008.

28. Bernal S, Calaf M, Garcia-Hoyos M, et al: Study of the involvement of the RGR, CRPB1, and CRB1 genes in the pathogenesis of autosomal recessive retinitis pigmentosa. J Med Genet 40: e89, 2003.

29. Henderson RH, Waseem N, Searle R, et al: An assessment of the apex microarray technology in genotyping patients with Leber congenital amaurosis and early-onset severe retinal dystrophy. Invest Ophthalmol Vis Sci 48: 5684-5689, 2007.

30. Simpson DA, Clark GR, Alexander S, et al: Molecular diagnosis for heterogeneous genetic diseases with targeted high-throughput DNA sequencing applied to retinitis pigmentosa. J Med Genet 48: 145-151, 2011.

31. Hanein S, Perrault I, Gerber S, et al: Leber congenital amaurosis: comprehensive survey of the genetic heterogeneity, refinement of the clinical definition, and genotype-phenotype correlations as a strategy for molecular diagnosis. Hum Mutat 23: 306-317, 2004

32. Wang Q, Wang P, Li S, et al: Mitochondrial DNA haplogroup distribution in Chaoshanese with and without myopia. Mol Vis 16: 303-309, 2010.

33. Nishiguchi KM and Rivolta C: Genes associated with retinitis pigmentosa and allied diseases are frequently mutated in the general population. PLoS One 7: e41902, 2012.

34. Jiao X, Wang P, Li S, et al: Association of markers at chromosome 15 q14 in Chinese patients with moderate to high myopia. Mol Vis 18: 2633-2646, 2012.

35. Tosi J, Tsui I, Lima LH, et al: Case report: autofluorescence imaging and phenotypic variance in a sibling pair with early-onset retinal dystrophy due to defective CRB1 function. Curr Eye Res 34: 395-400, 2009.

36. Li L, Xiao X, Li S, et al: Lack of phenotypic effect of triallelic variation in SPATA7 in a family with Leber congenital amaurosis resulting from CRB1 mutations. Mol Vis 17: 3326-3332, 2011.

37. Chen Y,Zhang Q, Shen T, et al: Comprehensive mutation analysis by whole-exome sequencing in 41 Chinese families with Leber congenital amaurosis. Invest Ophthalmol Vis Sci 54: 4351-4357, 2013.

38. Beryozkin A, Zelinger L, Bandah-Rozenfeld D, et al: Mutations in CRB1 are a relatively common cause of autosomal recessive early-onset retinal degeneration in the Israeli and Palestinian populations. Invest Ophthalmol Vis Sci 54: 2068-2075, 2013. 